\title{
Utilization of Medical Codes for Hypotension in Shock Patients: A Retrospective Analysis
}

\author{
Charles Hunley' \\ Shannon ME Murphy ${ }^{2}$ \\ Michael Bershad ${ }^{2}$ \\ Halit O Yapici ${ }^{3}$ \\ 'Department of Critical Care Medicine, \\ Orlando Regional Medical Center, \\ Orlando, FL, USA; ${ }^{2}$ Edwards Lifesciences, \\ Irvine, CA, USA; ${ }^{3}$ Boston Strategic \\ Partners, Inc., Boston, MA, USA
}

Correspondence: Charles Hunley Department of Critical Care Medicine, Orlando Regional Medical Center, 86

West Underwood Street, Suite 102,

Orlando, FL, 32806, USA

Tel +| 4079277955

Fax +I 32I 8433569

Email charles.hunley@orlandohealth.com
Purpose: To evaluate the utilization of hypotension diagnosis codes by shock type and year in known hypotensive patients.

Patients and Methods: Retrospective analysis of the Medicare fee-for-service claims database. Patients with a shock diagnosis code between 2011 and 2017 were identified using the International Classification of Diseases, Ninth and Tenth Revision, Clinical Modification (ICD-9-CM and ICD-10-CM). Based on specific ICD codes corresponding to each shock type, patients were classified into four mutually exclusive cohorts: cardiogenic shock, hypovolemic shock, septic shock, and other/unspecified shock. Annual proportion and counts of cases with at least one hypotension ICD code for each shock cohort were generated to produce 7-year medical code utilization trends. A Cochran-Armitage test for trend was performed to evaluate the statistical significance.

Results: A total of 2,200,275 shock patients were analyzed, 13.3\% ( $\mathrm{n}=292,192)$ of which received a hypotension code. Hypovolemic shock cases were the most likely to receive a hypotension code $(18.02 \%, n=46,544)$, while septic shock cases had the lowest rate $(11.48 \%, \mathrm{n}=158,348)$. The proportion of patients with hypotension codes for other cohorts were $18.0 \%(n=46,544)$ for hypovolemic shock and $16.9 \%(n=32,024)$ for other/unspecified shock. The presence of hypotension codes decreased by $0.9 \%$ between 2011 and 2014, but significantly increased from $10.6 \%$ in 2014 to $17.9 \%$ in 2017 ( $\mathrm{p}<0.0001, \mathrm{Z}=-105.05$ ).

Conclusion: Hypotension codes are remarkably underutilized in known hypotensive patients. Patients, providers, and researchers are likely to benefit from improved hypotension coding practices.

Keywords: blood pressure, clinical coding, data accuracy, International Classification of Diseases

\section{Introduction}

Hypotension can be described as decreased blood pressure below accepted values. In the absence of a universal definition, clinical guidelines describe hypotension as systolic blood pressure below $90-\mathrm{mmHg}$ or mean arterial pressure (MAP) below 65-mmHg. ${ }^{1}$ Hypotension is the main component of shock, a life-threatening condition of circulatory failure characterized by decreased oxygen delivery and/or increased oxygen consumption. Shock manifests with hypotension and may lead to tissue and cellular hypoxia. ${ }^{2}$

Exposure to hypotension is associated with adverse patient outcomes. A prospective cohort study from a large urban emergency department found an $18 \%$ increase in mortality for hypotensive versus non-hypotensive patients. ${ }^{3}$ A systematic review comprised of 42 high-quality studies agreed that prolonged 
exposure to hypotension increased the risk for severe kidney injury, myocardial injury, and stroke. $^{4}$ Furthermore, a study reported that delayed medical emergency team reviews for hypotensive patients were associated with significantly increased mortality, which suggests timely measurement and tracking of hypotension may lead to improved patient outcomes. ${ }^{5}$

Appropriate documentation of medical records is important to ensure coordination among healthcare providers and accurate provider payments. ${ }^{6,7}$ Detailed medical documentation and accurate coding may improve patient care by communicating comprehensive information to the clinicians who provide the subsequent care. ${ }^{7}$ Furthermore, accurate coding is necessary for appropriate and timely claims payments for hospitals and physicians. ${ }^{7}$ When health conditions are not coded or coded incorrectly, the result is an increase in aggregate healthcare system costs and decreased provider reimbursement. A 2002 study estimated that $6.3 \%$ of Medicare spending was improperly paid due to errors in medical coding, ${ }^{8}$ and recent estimates show that $75 \%$ to $80 \%$ of medical bills contain inaccurate charges due to errors in medical coding. ${ }^{9}$

Quality initiatives and retrospective research are dependent upon accurate medical coding, and the utility of claims data is increasing as an efficient, reliable, and inexpensive resource in health services research. ${ }^{10}$ Recent quality-of-care initiatives by the Centers for Medicare and Medicaid Services (CMS) heavily rely on the data collected/recorded by clinicians, and the demand for accurate data is likely to increase with a shift towards value-based reimbursement models. ${ }^{11}$ CMS recently adopted a quality measure evaluating hypotension, which will likely increase the importance of appropriate documentation in hypotensive patients. ${ }^{12}$ Furthermore, epidemiological research utilizing claims datasets is crucial to identify national burden/incidence trends over time for various diseases. $^{13,14}$

Despite its real-world implications, medical coding is a vastly understudied area. To our knowledge, there are no studies investigating medical coding in hypotensive patients. Herein, we present an evaluation of hypotension code utilization and coding trends for known shock patients.

\section{Patients and Methods}

\section{Data Source}

We conducted a retrospective, observational study using the $100 \%$ Medicare fee-for-service claims database
(Woodlawn, Maryland, US), which contains physician and hospital reimbursement information for the US population aged $\geq 65$-years as well as beneficiaries with certain disabilities (ie end-stage renal disease). Medicare fee-forservice database contains de-identified Health Insurance Portability and Accountability Act (HIPAA) compliant data and therefore is exempt from the need for a review by an institutional review board.

\section{Study Population}

Based on the International Classification of Diseases, Ninth and Tenth Revision, Clinical Modification (ICD9-CM and ICD-10-CM) Systems, the study included patients who received a shock diagnosis code (ICD-9: 785.50, 785.51, 785.52, 785.59; ICD-10: R57.0, R57.1, R57.8, R57.9, R65.21) between 2011 and 2017. Cases with orthostatic hypotension (458, I95.1), chronic hypotension (458.1), and hypotension induced by drugs (I95.2) or hemodialysis $(458.21,195.3)$ were excluded from the study. Patients were classified into four mutually exclusive cohorts based on shock type using ICD-9 and -10 codes: cardiogenic shock (785.51, R57.0), hypovolemic shock (785.59, R57.1), septic shock (785.52, R65.21), and other/unspecified shock (785.50, R57.8, R57.9).

\section{Outcomes and Analyses}

We calculated the proportion and counts of cases with at least one hypotension code (ICD-9: 458.29, 458.8, 458.9; ICD-10: I95.0, I95.81, I95.89, I95.9) from 2011 to 2017 for each shock cohort. Descriptive statistics, as well as $t$-tests and chi-squared tests, were used to characterize variation in patient demographics (ie age, sex, race, region) based on the presence of a hypotension code across each shock cohort. We also investigated the 7-year trends in hypotension and shock code utilization. A Cochran-Armitage test was performed to evaluate the statistical significance of the trend in hypotension code use.

\section{Results}

\section{Patient Characteristics}

A total of 2,200,275 patients met study inclusion and exclusion criteria and were divided into four cohorts based upon the shock type. Septic shock was the largest cohort $(n=1,379,564,63 \%)$, and other/unspecified shock was the smallest cohort ( $\mathrm{n}=189,791,9 \%)$ (Figure 1). The average age (standard deviation) for all patients was 72.2 


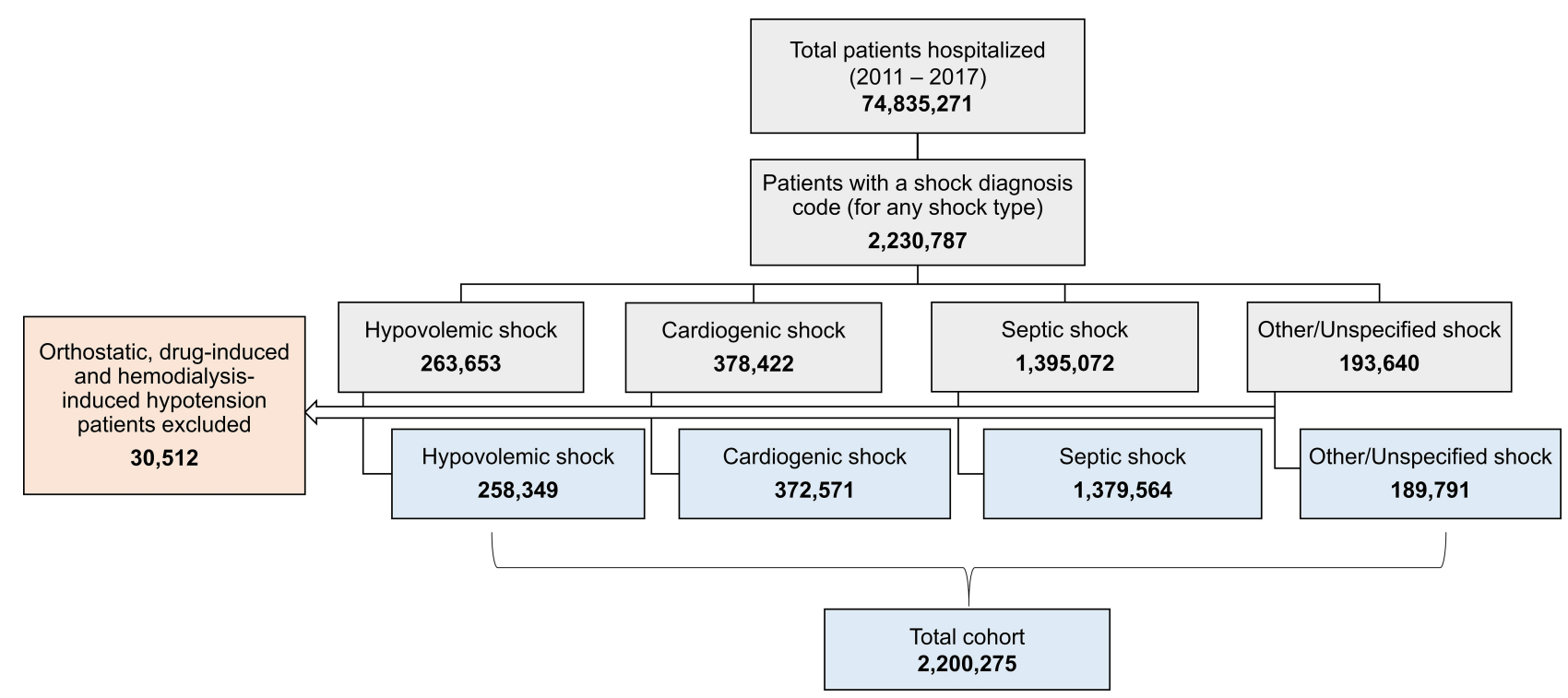

Figure I Patient attrition diagram.

Note: The patient counts included in the study and within each cohort are in bold for emphasis.

(12.5) years; $47.9 \%$ were female, and $81.0 \%$ were Caucasian. The majority of patients received care in the South $(40.6 \%)$ or Midwest $(22.9 \%)$ census regions of the US. Detailed demographic characteristics of study cohorts are provided in Table 1.

\section{Hypotension Code Utilization}

The counts and proportion of shock cases with at least one hypotension code, by shock type, are presented in Table 2. Of the 2,200,275 shock cases, $13.3 \%(n=292,192)$ received at least one hypotension code. Hypotension codes were included in claims for $18.0 \%(n=46,544)$ of hypovolemic shock cases, $16.9 \%(n=32,024)$ of other/unspecified shock cases, $14.8 \%(n=55,276)$ of cardiogenic shock cases, and $11.5 \%(n=158,348)$ of septic shock cases.

\section{Code Utilization Trends 20II-2017}

Shock and hypotension code utilization trends are illustrated in Figure 2. Hypotension code utilization for shock patients decreased initially from $11.5 \%$ (2011) to $10.6 \%$ (2014), however, it increased in each subsequent year to $17.9 \%$ by 2017 ( $\mathrm{p}<0.0001, \mathrm{Z}=$ -105.0520; years 2011-2017). The surge in hypotension code utilization was significant for all shock types based on a Cochran-Armitage trend test (Table 3). The presence of shock codes trended upwards, with an $18.9 \%$ overall increase in the number of reported cases between 2011 $(n=286,354)$ and $2017(n=340,544)$.

\section{Discussion}

This analysis revealed an overall low utilization of hypotension codes among known hypotensive cases. Only $13.3 \%$ of all shock cases included a hypotension code. The lack of a single, standardized definition of hypotension may partly explain the underutilization of these codes. ${ }^{15}$ Clear guidance and thresholds regarding the diagnosis of hypotension might increase the use of appropriate diagnosis codes.

Despite being the most prevalent shock type, the septic shock had the lowest hypotension code rate. The difference between septic shock and other shock types can be partially explained by the Surviving Sepsis guidelines, which provides a detailed evaluation of sepsis, including a MAP $<65-\mathrm{mmHg}$ threshold for diagnosing septic shock. ${ }^{16}$ Physicians may be documenting hypotension relatively less in septic shock patients due to the MAP criterion in the diagnosis of septic shock. In contrast, the hypotensive patients with other shock types, which lack a similar guideline, may be more likely to receive a separate hypotension code. However, the differences among shock diagnosis guidelines (ie the presence of MAP criteria) do not explain the overall low utilization for hypotension across shock types.

We showed a significant increasing trend in hypotension code utilization, most notably after 2014, while the presence of the shock codes consistently increased across all study years (2011 to 2017). The increase in the presence of both hypotension and shock codes after 2014 


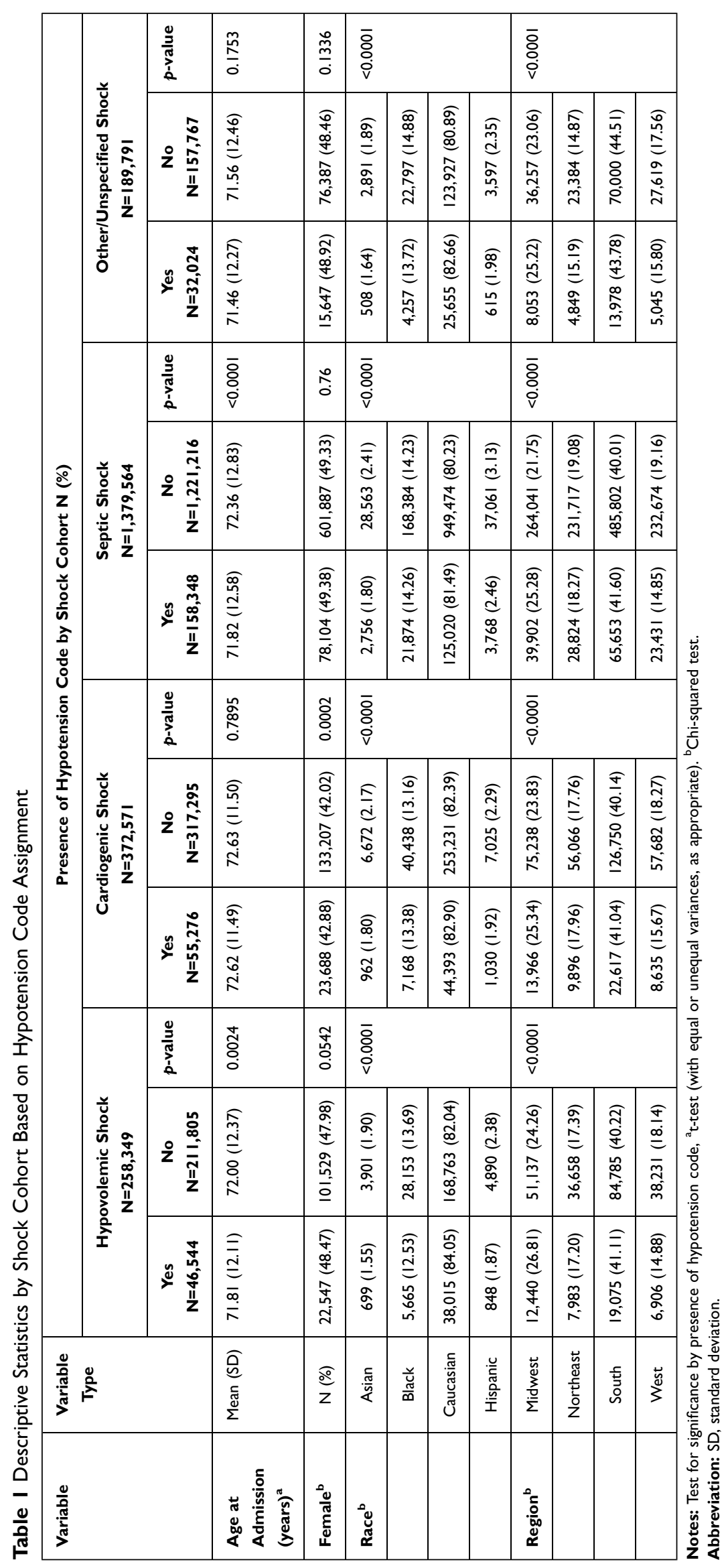


Table 2 Counts and Percentages of Cases with Hypotension Codes by Shock Type

\begin{tabular}{|l|c|c|c|}
\hline Description & Total Cases & \multicolumn{2}{|c|}{$\begin{array}{c}\text { Cases with } \\
\text { a Hypotension Code }\end{array}$} \\
\cline { 3 - 4 } & & Counts & Percentage \\
\hline Hypovolemic Shock & 258,349 & 46,544 & $18.02 \%$ \\
Cardiogenic Shock & $372,57 \mid$ & 55,276 & $14.84 \%$ \\
Septic Shock & $1,379,564$ & 158,348 & $11.48 \%$ \\
Other/Unspecified Shock & 189,791 & 32,024 & $16.87 \%$ \\
Total & $2,200,275$ & 292,192 & $13.28 \%$ \\
\hline
\end{tabular}

overlaps with the transition from ICD-9-CM to ICD-10$\mathrm{CM}$ coding systems, which likely improved overall coding practices. The 2012 Surviving Sepsis Guidelines boosted shock awareness considerably among the medical community and may have contributed to the isolated increase in shock code utilization before 2014. Efforts to increase awareness and education among clinicians and medical coders may lead to increased use of appropriate codes. ${ }^{17}$

Exposure to hypotension is harmful to patients and may lead to significant mortality and morbidity. ${ }^{3,4}$ Accurate inclusion of hypotension in medical records may have positive downstream impacts on patient care by supporting quality monitoring/improvement initiatives as well as informing program development and patient identification for payerbased population health initiatives (ie care management). ${ }^{18}$ The importance of record-keeping is likely to increase as CMS is currently testing the submission of quality measure data through electronic health records (EHR). ${ }^{11}$ A quality measure evaluating hypotension was recently adopted by
CMS, which will impact payments to providers in the Meritbased Incentive Payment System. ${ }^{12}$ As payers increase data requirements from the providers, clear documentation of major and comorbid conditions may have a more significant impact in the future pay-for-performance programs. ${ }^{7}$ Clearly defined hypotension codes could reduce the burden of reporting.

Accurate coding of hypotension may lead to higher quality retrospective research and improved accuracy for a national burden estimate, which could increase funding for research, education, and treatment for hypotensive patients. ${ }^{13}$ Despite its widely-known shortcomings (ie delay in availability, limited clinical information, sensitivity), claims data is increasingly being used in health services research as an efficient and relatively low-cost data source. $^{10,19-21}$ Moreover, claims databases provide a combination of clinical and reimbursement-related information for almost all health encounters, which is especially beneficial when high-volume and long-term follow-up is important to the research. ${ }^{19}$ Information from the claims data may also be used to complement other data. The Food and Drug Administration previously established national strategies by combining information from registries, EHR, and claims databases. ${ }^{19}$ Furthermore, Hlatky et al and Brennan et al suggested that claims data can be used to evaluate outcomes of participants with reasonable accuracy in later phases of large pragmatic clinical trials and medical device surveillance systems, respectively. ${ }^{19,20}$

Our results suggest a remarkably low use of hypotension codes among known hypotensive patients and differing rates

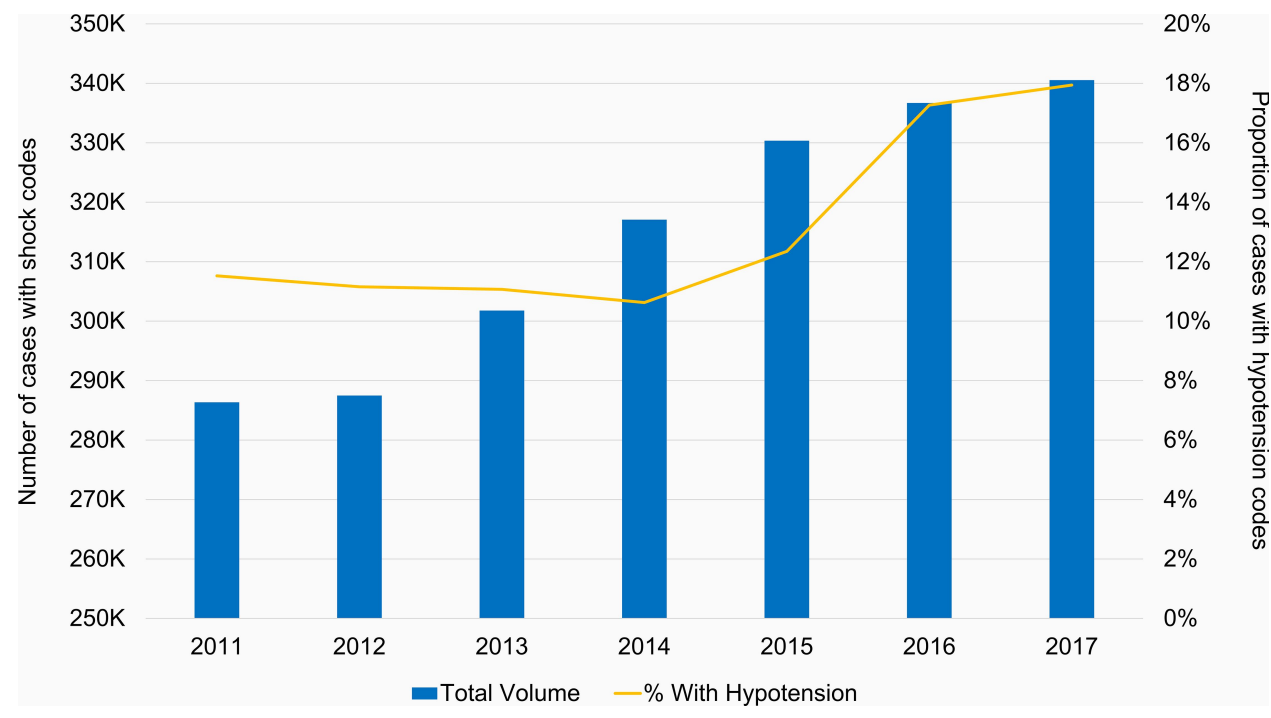

Figure 2 Seven-year trends in the shock and hypotension diagnosis code utilization. 
Table 3 Cochran-Armitage Trend Test Analysis by Shock Cohort Between 201I and 2017

\begin{tabular}{|c|c|c|c|c|c|c|c|c|c|}
\hline \multirow[t]{2}{*}{ Shock Cohort } & \multicolumn{7}{|c|}{$\begin{array}{l}\text { Percentages of Shock Cases with Hypotension Codes by } \\
\text { Year }\end{array}$} & \multirow[t]{2}{*}{$\begin{array}{l}\text { Cochran-Armitage Test } \\
\text { Statistic }\end{array}$} & \multirow[t]{2}{*}{$p$-value } \\
\hline & 2011 & 2012 & 2013 & 2014 & 2015 & 2016 & 2017 & & \\
\hline Hypovolemic shock & $15.11 \%$ & $14.42 \%$ & $14.80 \%$ & $14.02 \%$ & $16.47 \%$ & $27.35 \%$ & $28.28 \%$ & -58.0582 & $<0.0001$ \\
\hline Cardiogenic shock & $12.78 \%$ & $12.50 \%$ & $12.34 \%$ & $11.53 \%$ & $13.03 \%$ & $20.02 \%$ & $20.68 \%$ & -49.4383 & $<0.0001$ \\
\hline Septic shock & $10.15 \%$ & $9.77 \%$ & $9.60 \%$ & $9.23 \%$ & $10.73 \%$ & $14.70 \%$ & $15.24 \%$ & -71.3401 & $<0.0001$ \\
\hline Other/Unspecified shock & $14.52 \%$ & $14.06 \%$ & $13.87 \%$ & $13.88 \%$ & $16.97 \%$ & $20.06 \%$ & $20.32 \%$ & -29.2167 & $<0.0001$ \\
\hline
\end{tabular}

by shock types. Despite current studies suggesting significant real-world implications of inappropriate medical coding practices, the specific impact of underutilization of diagnosis codes on patient care, financial efficiency, and quality of retrospective research is yet to be studied.

\section{Limitations}

A strength of this study is the large sample size; however, it is not without limitations. As previously discussed, claims databases are designed for administrative purposes and have inherent limitations regarding retrospective research. There are restrictions on the number of diagnosis codes that are recorded on claims forms; therefore, our study may have underestimated the shock/hypotension code utilization, particularly given the older population present in the Medicare claims database, which is more likely to receive multiple diagnosis codes corresponding to various comorbid conditions. Additionally, as previously shown by Menon et al, a small yet significant subset of cardiogenic shock patients (5\%) may present with hypoperfusion but not hypotension, or what is known as non-hypotensive cardiogenic shock. ${ }^{22}$

\section{Conclusion}

Hypotension codes appear to be underutilized in known hypotensive patients. Establishing clear guidelines to identify and classify hypotension, as well as improving education/training for clinicians and medical coders, is likely to improve coding practices, which has potential benefits for patients, providers, and the research community. Accurate coding is likely to improve health services research and quality initiatives, which may consequently improve patient outcomes. Further research is required to establish the causal relationship between the utilization of hypotension codes and improvements in patient outcomes.

\section{Abbreviations}

MAP, mean arterial pressure; CMS, Centers for Medicare and Medicaid Services; HIPAA, Health Insurance Portability and Accountability Act; ICD-9-CM, International Classification of Diseases Ninth Revision Clinical Modification; ICD-10$\mathrm{CM}$, International Classification of Diseases Tenth Revision Clinical Modification; EHR, electronic health records.

\section{Acknowledgments}

The authors would also like to acknowledge Ms. Auma Muttu and Ms. Anjani R. Parikh from Boston Strategic Partners, Inc. (supported by Edwards Lifesciences) for their editorial support.

\section{Funding}

This study was funded by Edwards Lifesciences.

\section{Disclosure}

Dr. Hunley received consulting fees from Edwards Lifesciences. Dr. Bershad and Ms. Murphy are employees of Edwards Lifesciences. Dr. Yapici is an employee of Boston Strategic Partners, Inc., which received funds from Edwards Lifesciences for research and editorial support. The authors report no other conflicts of interest in this work.

\section{References}

1. Sharma S, Hashmi MF, Bhattacharya PT. Hypotension: StatPearls; 2020.

2. Haseer Koya H, Paul M. Shock: StatPearls; 2020.

3. Jones AE, Stiell IG, Nesbitt LP, et al. Nontraumatic out-of-hospital hypotension predicts inhospital mortality. Ann Emerg Med. 2004;43 (1):106-113. doi:10.1016/j.annemergmed.2003.08.008

4. Wesselink EM, Kappen TH, Torn HM, Slooter AJC, van Klei WA. Intraoperative hypotension and the risk of postoperative adverse outcomes: a systematic review. Br J Anaesth. 2018;121(4):706-721. doi:10.1016/j.bja.2018.04.036

5. Quach JL, Downey AW, Haase M, Haase-Fielitz A, Jones D, Bellomo R. Characteristics and outcomes of patients receiving a medical emergency team review for respiratory distress or hypotension. J Crit Care. 2008;23(3):325-331. doi:10.1016/j.jcrc. 2007.11.002 
6. DeParle N-A. Evaluation \& Management Services Guidelines. JAMA. 2000;283(23):3061. doi:10.1001/jama.283.23.3061

7. McNally $M$ The importance of detailed documentation in ICD-10. American College of Surgeons. Available from: https://bulletin.facs. org/2015/08/the-importance-of-detailed-documentation-in-icd-10/. Accessed January 13, 2020.

8. Adams DL, Norman H, Burroughs VJ. Addressing medical coding and billing part II: a strategy for achieving compliance. A risk management approach for reducing coding and billing errors. J Natl Med Assoc. 2002;94(6):430-447.

9. Study shows improper EHR medical coding cause inaccurate medical bills and lost revenue; EHR shortcuts to blame. Press Release. PRWeb; 2017. Available from: https://www.prweb.com/releases/ 2017/06/prweb14459212.htm. Accessed March 26, 2021.

10. Psaty BM, Delaney JA, Arnold AM, et al. Study of Cardiovascular Health Outcomes in the Era of Claims Data: the Cardiovascular Health Study. Circulation. 2016;133(2):156-164. doi:10.1161/ CIRCULATIONAHA.115.018610

11. Core Measures. Centers for medicare \& medicaid services. Available from: https://www.cms.gov/Medicare/Quality-Initiatives-PatientAssessment-Instruments/QualityMeasures/Core-Measures. Accessed January 13, 2020.

12. Centers for Medicare and Medicaid Services. 2020 QCDR Measure Specifications. Updated August 3, 2020. Available from: https://ppcm-prod-content.s3.amazonaws.com/uploads/804/2020_QCDR_ Measure_Specifications_v2.0.xlsx. Accessed December 14, 2020.

13. Deshayes S, Moulis G, Pillebout E, Aouba A, Audemard-Verger A. Positive predictive value of hospital discharge diagnosis code to identify immunoglobulin A vasculitis in France: a validation study. Eur J Intern Med. 2017;43:e18-e19. doi:10.1016/j.ejim.2017.05.025

14. Andes LJ, Li Y, Srinivasan M, Benoit SR, Gregg E, Rolka DB. Diabetes Prevalence and Incidence Among Medicare Beneficiaries United States, 2001-2015. MMWR Morb Mortal Wkly Rep. 2019;68 (43):961-966. doi:10.15585/mmwr.mm6843a2
15. Bijker JB, van Klei WA, Kappen TH, van Wolfswinkel L, Moons KG, Kalkman CJ. Incidence of intraoperative hypotension as a function of the chosen definition: literature definitions applied to a retrospective cohort using automated data collection. Anesthesiology. 2007;107(2):213-220. doi:10.1097/01.anes.0000270 724.40897.8e

16. Plevin R, Callcut R. Update in sepsis guidelines: what is really new? Trauma Surg Acute Care Open. 2017;2(1):e000088. doi:10.1136/ tsaco-2017-000088

17. Watzlaf V, Alkarwi Z, Meyers S, Sheridan P. Physicians' Outlook on ICD-10-CM/PCS and Its Effect on Their Practice. Perspect Health Inf Manag. 2015;12(Winter): $1 \mathrm{~b}$.

18. Kan HJ, Kharrazi H, Leff B, et al. Defining and assessing geriatric risk factors and associated health care utilization among older adults using claims and electronic health records. Med Care. 2018;56 (3):233-239. doi:10.1097/MLR.0000000000000865

19. Brennan JM, Wruck L, Pencina MJ, et al. Claims-based cardiovascular outcome identification for clinical research: results from 7 large randomized cardiovascular clinical trials. Am Heart J. 2019;218:114. doi:10.1016/j.ahj.2019.09.002

20. Hlatky MA, Ray RM, Burwen DR, et al. Use of Medicare data to identify coronary heart disease outcomes in the Women's Health Initiative. Circ Cardiovasc Qual Outcomes. 2014;7(1):157-162. doi:10.1161/CIRCOUTCOMES.113.000373

21. Ollendorf DA, Fendrick AM, Massey K, Williams GR, Oster G. Is sepsis accurately coded on hospital bills? Value Health. 2002;5 (2):79-81. doi:10.1046/j.1524-4733.2002.52013.x

22. Menon V, Slater JN, White HD, Sleeper LA, Cocke T, Hochman JS. Acute myocardial infarction complicated by systemic hypoperfusion without hypotension: report of the SHOCK trial registry. Am J Med. 2000;108(5):374-380. doi:10.1016/s0002-9343(00)00310-7
Journal of Multidisciplinary Healthcare

\section{Publish your work in this journal}

The Journal of Multidisciplinary Healthcare is an international, peerreviewed open-access journal that aims to represent and publish research in healthcare areas delivered by practitioners of different disciplines. This includes studies and reviews conducted by multidisciplinary teams as well as research which evaluates the results or conduct of such teams or healthcare processes in general. The journal

Submit your manuscript here: https://www.dovepress.com/journal-of-inflammation-research-journal covers a very wide range of areas and welcomes submissions from practitioners at all levels, from all over the world. The manuscript management system is completely online and includes a very quick and fair peer-review system. Visit http://www.dovepress.com/testimonials. php to read real quotes from published authors. 